Original Papers

\title{
Construction of Proton Nuclear Magnetic Resonance Parameter Database System
}

\author{
Osamu Yamamoto, Kikuko Hayamizu and Masaru Yanagisawa \\ National Chemical Laboratory for Industry, Tsukuba, Ibaraki 305 \\ O.Y.: Present address Kanda University for International Studies, Wakaba, Chiba 260
}

\begin{abstract}
A IH-NMR database comprised of NMR parameters, i.e., the chemical shifts and the coupling constants, is described. The data are compiled from the literature after full evaluation as well as from the data obtained in our laboratory, and registered to a master file, from which an access file for users is made. Spectral patterns are generated by a calculation using the parameters in the database on user's request. The calculation program and the procedure for extraction of the parameters are discussed. Advantages and limitations of the parameter database are also pointed out.
\end{abstract}

Keywords Spectral database, proton nuclear magnetic resonance parameters

As discussed in our previous paper ${ }^{1}$, the utility of the ${ }^{1}$ H-NMR spectral database in the identification of unknown compounds by a pattern matching method is limited due to the frequency dependence of ${ }^{1} \mathrm{H}-\mathrm{NMR}$ spectral patterns, so that the comparison of an unknown spectrum with reference data is possible only for the spectra observed at the same resonance frequency. The best way to solve the difficulty is to create a ${ }^{1} \mathrm{H}-\mathrm{NMR}$ parameter database which is comprised of both chemical shifts and coupling constants. In the parameter database system, spectral patterns at any resonance frequency can be generated by a well-known quantum mechanical-calculation, so that the limitation due to the frequency dependence of the spectral patterns can be removed.

Another advantage of the parameter database is that the memory size can be greatly reduced. While at least 5-10 Kbytes are necessary for a spectrum in the pattern database $^{1}$, the memory size of one spectrum required for the parameter database will be less than $1 \mathrm{~K}$ byte even when the compound name or the molecular formula and the sample conditions are included.

The advantages of the parameter database are evident, but it is also clear that the creation of the parameter database is a tedious project. In general, the extraction of the 'H-NMR parameters from a given spectrum is not easy, especially for the spectra in which many spins are strongly coupled with each other. The ${ }^{1} \mathrm{H}-\mathrm{NMR}$ parameters have been reported in a great many papers and summarized in a handbook by Brügel $^{2}$, but the number of the compounds is not enough to cover representative organic compounds.

Nevertheless, it is worthwhile to have a ${ }^{1} \mathrm{H}$ parameter database, since it can play an important role to supplement the ${ }^{1} \mathrm{H}$ pattern database. Thus, we have constructed a ${ }^{1} \mathrm{H}$ parameter database as a subsystem in the ${ }^{1} \mathrm{H}-\mathrm{NMR}$ database which is, in turn, one of subsystems of the integrated spectral database system named SDBS for IR, MS, ${ }^{1} \mathrm{H}-\mathrm{NMR},{ }^{13} \mathrm{C}-\mathrm{NMR}$, Raman and ESR spectra. ${ }^{3}$ In this paper, we present the outline of the ${ }^{1} \mathrm{H}$ parameter database subsystem.

\section{Results and Discussion}

The 'H-NMR database in SDBS has two subsystems, one of which is for the pattern datal and the other for the parameter data. The data for the parameter database are collected in the following two ways:

(1) In the course of the compilation of ${ }^{1} \mathrm{H}-\mathrm{NMR}$ spectra observed in our laboratory into the pattern database, if the spectrum shows a simple pattern, i.e., it consists of a few single lines with no coupling, or shows a typical coupling pattern from which the NMR parameters can be extracted easily, the spectrum is compiled into the parameter database after extracting the NMR parameters from the spectrum.

(2) The parameters are taken from the literature after full evaluation of the data. The criteria are as follows: (a) All the ${ }^{1} \mathrm{H}-\mathrm{NMR}$ parameters in a molecule should be listed in the literature. When a molecule contains one or more abundant nuclear spins other than ${ }^{1} \mathrm{H}$ such as ${ }^{19} \mathrm{~F}$ and ${ }^{31} \mathrm{P}$, the spin coupling constants between protons and such spins should be included. (b) The chemical shift values in ppm should be reported to at least two digits beyond the decimal point, and the 
coupling constants in $\mathrm{Hz}$ to at least one digit beyond the decimal point, with few exceptions. (c) When several sets of the parameters are reported in various papers for the same compound, more recent values with higher precision are to be taken. In many cases in the literature, the most precise values of the coupling constants were determined in the neat liquid. Generally the coupling constants for molecules not undergoing chemical exchange, are independent of the sample conditions such as the solvent, the concentration, and the measuring temperature. When the chemical shift values in a diluted solution are reported, but some coupling constants such as small long-range coupling constants are not described, we combined the chemical shifts in a diluted concentration and the coupling constants in the neat liquid. The number of such cases is not large and a comment is given in the data of the database system. For the collection of the data from the literature, at first we consulted Brügel's handbook ${ }^{2}$, and then referred to the original papers. Since the handbook covers the literature until 1976, we collected data after that mainly from the papers in the journal: Magnetic Resonance in Chemistry. About $25 \%$ of the compounds summarized in the handbook were adopted in our parameter database, and the remaining data do not satisfy the criteria mentioned above. One of the main reasons why the data were not adopted is the lack of the complete sets of the parameters. For example, in methylnaphthalenes the chemical shift values of the methyl protons were not listed in the original paper. ${ }^{4}$

\section{Data File Structure}

The data files of our ${ }^{1} \mathrm{H}-\mathrm{NMR}$ parameter subsystem consist of a MASTER FILE and an ACCESS FILE. MASTER FILE has a similar structure to that of MASTER FILE I of the pattern subsystem ${ }^{1}$ except that it also contains the spectral information, i.e., the ${ }^{1} \mathrm{H}-$ NMR parameters.

The input to MASTER FILE can be carried out manually in a similar manner to that used with MASTER FILE I of the pattern subsystem. MASTER FILE includes the records given below, for which similar items have similar meanings as in the pattern subsystem.

Record 1: Spectral key to each spectrum.

Record 2: Structural formula with spectral assignments.

Record 3: Quality level of the spectral data. In the parameter subsystem, the level is determined by the precision of the parameter values.

Record 4: Conditions of the sample.

Record 5: Number of independent spin blocks, $\mathrm{N}$, and their intensity factors. In an independent spin block the spins in a block do not couple with spins in other block(s) in a molecule.

Record 6: NN, ISO and HETERO in a spin block. $\mathrm{NN}$ means the number of non-equivalent spin groups. e.g., $\mathrm{NN}=2$ for $\mathrm{CH}_{3} \mathrm{CH}_{2} \mathrm{Cl}$ which gives a spectrum of an $A_{3} B_{2}$ spin system, if the free rotation of the methyl group is assumed. ISOs the symbols showing the assignments of the spins such as A and $B$ in the above example, and HETERO the information on the abundant magnetic nuclei other than protons.

Record 7: NUCL, FR1, FR2, AMIN, T2 in a spin block. NUCLs are numbers of spins in each nonequivalent spin group (in the above example, they are 3 and 2). FR1 and FR2 define the range of frequencies for the calculation, AMIN is the minimum intensity taken into account, and T2s are the line widths for the spins.

Record 8: Chemical shift values in ppm for each non-equivalent spin group.

Record 9: Coupling constants in $\mathrm{Hz}$ between each non-equivalent spin group.

Record 6-9 are repeated for every set of the independent spin blocks (number of the sets is shown in N).

Record 10: Origin of the parameters for a compound. The references are included.

Record 11: Comment, if any. The most important comments are concerned with the parameters about the satellites from ${ }^{13} \mathrm{C},{ }^{29} \mathrm{Si},{ }^{11} \mathrm{~B},{ }^{117} \mathrm{Sn},{ }^{119} \mathrm{Sn},{ }^{119} \mathrm{Hg}$ and other magnetic nuclei. The ${ }^{19} \mathrm{~F}$ and ${ }^{31} \mathrm{P}$ chemical shift values are also included if they were found in the references. Sometimes long range-coupling constants are noted in a manner such as being less than $0.2 \mathrm{~Hz}$.

An example of the input data to EDITOR ${ }^{1}$ is shown in Fig 1. The data from the EDITOR are compressed and registered to MASTER FILE in the parameter database. ACCESS FILE, the binary file for users, is made from MASTER FILE by a program.

\section{Generation of the Spectral Pattern}

The generation of the ${ }^{1} \mathrm{H}$ spectral pattern in our

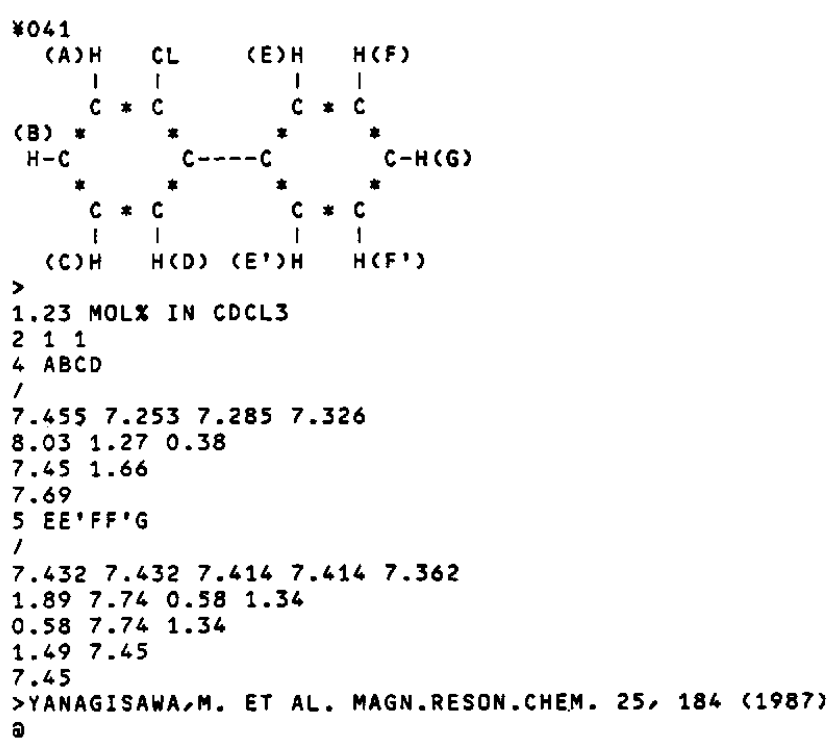

Fig. 1 An example of an entry of MASTER FILE in the EDITOR form. 
parameter subsystem is made by a FORTRAN program named LAOCNCLI. The original program for the calculation of the NMR spectra is LAOCN35, which was extended so as to take the concept of the magnetic equivalence factoring ${ }^{6}$ into account, and up to 5 non-equivalent spin groups can be treated in LAOCN-4A. ${ }^{7}$ We modified LAOCN-4A to be able to treat up to 9 spin groups and to divide the total spin system into independent spin blocks in LAOCNCLI. The most time-consuming part of this program is the diagonalization of the matrices of the spin Hamiltonian. We use a FACOM M780 as a main frame computer, and can generate a spectrum within the order of a turn-around time in a TSS mode.

Since the frequencies are obtained as differences of two large terms (the diagonalized energy level terms), it is preferable that the diagonalization of the energy matrices is made with double precision in a computer. This is particularly important for spin systems where some abundant magnetic nuclei other than protons are contained in a molecule. In the calculation, the resonance frequency of such hetero spins is offset from that of protons by $100 \mathrm{ppm}$ to assure the loose coupling between the hetero spins and protons, so that the energy level terms become too large to obtain the differences with accuracy of $0.1 \mathrm{~Hz}$, if the double precision calculation were not made.

When generating the spectral pattern, users can specify any resonance frequency for which a spectral pattern can be obtained. In addition to the standard option for generating and expanding the spectra in our SDBS, the ${ }^{1} \mathrm{H}-\mathrm{NMR}$ parameter subsystem has an option to change the NMR parameters. Since the line width and the chemical shift values are sensitive to the sample conditions, the user may slightly change such parameters, to see how the spectrum changes corresponding to the changes in the parameters. The user can thus determine the changes in the spectral pattern depending on the solvent, the concentration, the temperature and other factors.

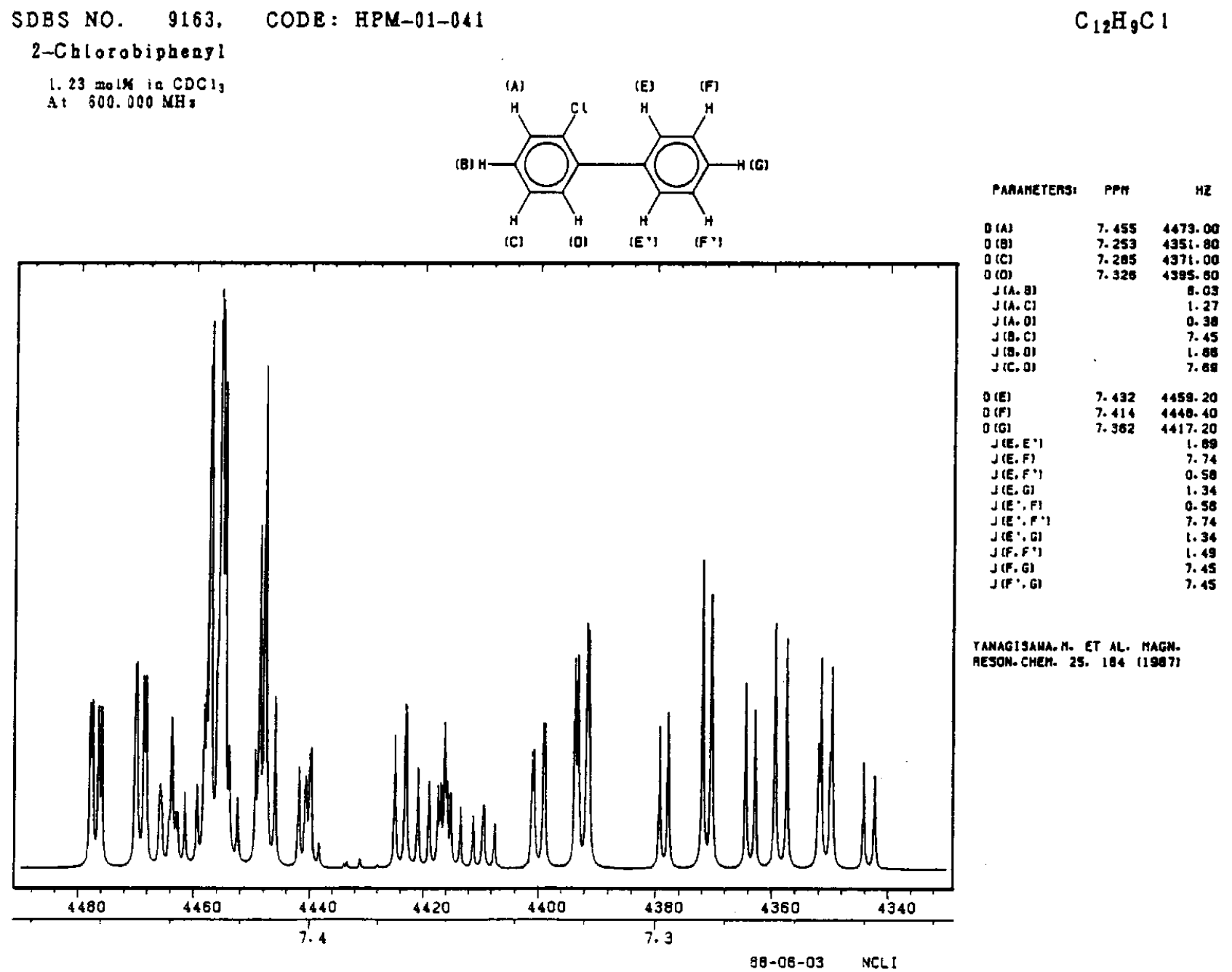

Fig. 2 The output of the ${ }^{1} \mathrm{H}-\mathrm{NMR}$ spectrum to a laser printer from the ${ }^{1} \mathrm{H}-\mathrm{NMR}$ parameter detabase. An example is a $600-\mathrm{MHz}$ spectrum of 2-chlorobiphenyl in $\mathrm{CDCl}_{3}$ solution, for which the $400-\mathrm{MHz}$ spectrum was observed and analyzed to extract the NMR parameters. 
As an example of an output of a spectrum from the ${ }^{1} \mathrm{H}$ parameter database, a $600-\mathrm{MHz}$ spectrum is generated for 2-chlorobiphenyl as shown in Fig. 2.

\section{Extraction of the NMR parameters}

As stated above, for creating the ${ }^{1} \mathrm{H}-\mathrm{NMR}$ parameter subsystem, we have collected the parameters by extracting from the spectra observed in our laboratory, as well as from the literature. Although most of them are taken from the spectra with rather simple patterns, the procedure used is rigorous, i.e, the iterative calculation by our LAOCNCLI program. The LAOCN program, in its generic sense, is directed to the extraction of NMR parameters by the least squares method rather than the simple generation of the spectral pattern. After the excellent work of BothnerBy and Castellano in the basic form ${ }^{5}$, many variations of the LAOCN program have been reported.

For the least squares calculation, the input of an observed spectrum with high quality is necessary. In LAOCN programs a set of trial values of the parameters are specified at first to generate a spectrum similar to the observed one. Next, the assignment of observed and the calculated lines is attempted, and then according to the assignment the trial values are corrected until a good convergence is obtained, by the least squares method using Jacobian matrices constructed from derivatives of the energy levels by the parameters. As usual in the least squares method, the calculation does not converge if the proper trial values were not selected. Furthermore, even if trial values are properly chosen, it is essential that the assignments of each observed line to the calculated one are interchanged to obtain more reliable values as the calculation proceeds. Thus it is preferable that the input data including the assignments can be easily changed in any stage of the calculation.

A shortcoming common to the LAOCN programs employed so far, seems to be inconvenience in changing the input data for the iterative calculation. In our modified LAOCNCLI program, all the data are input in menu formats from an character display in a TSS mode, and at the same time the data are saved in a dataset. In the course of the calculation the user may change any data at any stage by the menu. Thus the efficiency in the calculation greatly increases. The program is provided in SDBS as SIMULATE option. ${ }^{3}$

It is possible that the data in MASTER FILE II in the pattern subsystem can be directly input to LAOCNCLI program as an observed spectrum, although this option is only used for the purpose of constructing the parameter database efficiently, and is not opened to general users.

\section{Limitation of the ${ }^{1} H-N M R$ parameter database}

The usefulness of the ${ }^{1} \mathbf{H}-\mathrm{NMR}$ parameter database is clear from the discussion above. The reproducibility of spectra from the 'H-NMR parameters is in general excellent, as has been examined by many workers. The situations where this is not the case will be as follows: In the LAOCN calculation only the frequencies and intensities of the signals are obtained. The output of the spectral pattern is drawn somewhat arbitrarily by a Lorentzian line shape with suitable line widths. Strictly speaking, the spectral pattern is correct only when the widths of each line are the same. Even if the T2 values of each spin are specified, the line widths corresponding to mixed transitions are set only approximately in the program.

When some phenomena affecting the line shapes are present and the extremely narrowing condition is not satisfied due to the chemical exchange or other effects, the correct spectral pattern cannot be generated. In those cases, some other suitable method, e.g., the density matrix method is necessary. ${ }^{8-10}$ It should be noted that, in the chemically exchanging systems, the line widths are dependent on the observing resonance frequency.

Another problem is the long-range coupling. The line splittings less than ca. $0.3 \mathrm{~Hz}$ are not well resolved unless the sample is degassed. Small long-range coupling constants may not be obtained for an undegassed sample, especially for a complex spectrum. Therefore, the data in the parameter database are limited to the data where some long-range coupling could be assumed not to exist. During the evaluation procedure for the data in the literature, when the longrange coupling constants can be supposed to have small values, but they were not described, the data were not adopted, or the presence of possible long-range coupling constants is stated in the comment.

These limitations, however, can be removed in principle. Since the recent development in the NMR instruments, especially by introducing a superconducting magnet, allows us to measure a well resolved spectral pattern with better $S / N$ ratios and a narrower line widths. Then the extraction of the ${ }^{1} \mathbf{H}-\mathrm{NMR}$ parameters becomes easier than before. The data will be revised and replaced with more precise values. In future the procedure to generate the spectral pattern will not be limited to the LAOCN calculation, and will include the line shapes for the dynamic processes. Although it requires a long time to do, it will be the only way to create a good database.

At the end of May, 1988, the total number of the data in the ${ }^{1} \mathrm{H}-\mathrm{NMR}$ parameter database is about 2500. The number of the spectra whose parameters have been extracted from the spectral pattern in our laboratory is about 300 , approximately $7.5 \%$ of the ${ }^{1} \mathrm{H}$ spectra observed and registered in the ${ }^{1} \mathrm{H}-\mathrm{NMR}$ pattern database.

The authors express their hearty thanks to Ms. M. Itoh, Ms. T. Ishii and Ms. Y. Murata for their efforts in compiling the 'H-NMR parameters from the literature, extracting the parameters from the pattern data, writing the manuscripts and input of the data in constructing ${ }^{2} \mathrm{H}-\mathrm{NMR}$ parameter database. 


\section{References}

1. O. Yamamoto, K.Hayamizu and M. Yanagisawa, Anal. Sci., 4, 347 (1988).

2. W. Brügel, "Handbook of NMR Spectral Parameters", Vol, 1-3, Heyden, London (1979).

3. O. Yamamoto. K. Someno, N. Wasada, J. Hiraishi, K. Hayamizu. K. Tanabe, T. Tamura and M. Yanagisawa, Anal. Sci., 4, 233 (1988).

4. J. W. Emsley, R. R. Salman, and R. A. Storey, J. Chem. Soc., B 1970, 1513.

5. A. A. Bothner-By and S. M. Castellano in "Computer Programs for Chemistry", ed. D. F. DeTar, Vol. 1, p. 1, W. A. Benjamin, Inc., New York (1968), and " $L A O C N 3$ ",
QCPE NO. 111.

6. R. C. Fergason and D. W. Marquardt, J. Chem. Phys., 41, 2087 (1964).

7. J. A. Musso and A. Isaia, $N M R-L A O C N-4 A$ ", QCPE NO. 232.

8. C. S. Johnson, Jr., “Advances in Magentic Resonance”, ed. J. S. Waugh, Vol. 1, p. 33, Academic Press, New York (1965).

9. J. Sandstrom, "Dynamic NMR Spectroscopy", Academic Press, New York (1982).

10. D. S. Stephenson and G. Binsch, "DNMR5" QCPE No. 365.

(Received June 14, 1988)

(Accepted June 28, 1988) 